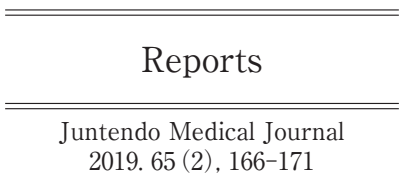

\title{
Report of the Tropical Medicine Association of Juntendo University's 54th South East Asia Research Group Activity and Prospects for Our Future Activities
}

\author{
SHOHEI FUJIKAWA*1) 9), TOMOKI HONGO*1) 9), HANA OTANI*2) 9), ChIKAKO NAOE*1) 9), \\ Megumi HISANAGA*3) 9), Shunya IIJIMA*4) 9), Kenta HIKOTANI*4) 9), RIHO TAKEDA*4) 9), \\ Momoko IIJIMA*4) 9), SAyUki OCHIAI*4) 9), KenJI MURAI*5) 9), TOSHIHIRO MITA*6) 9), \\ KENJI SUZUKI*7) 9), KAZUHISA TAKAHASHI*8) 9) \\ *1) The 4th Year Students of Faculty of Medicine, Juntendo University, Tokyo, Japan, *2) The 5th Year Students of Faculty \\ of Medicine, Juntendo University, Tokyo, Japan, *3) The 3rd Year Students of Faculty of Medicine, Juntendo University, \\ Tokyo, Japan, *4) The 2nd Year Students of Faculty of Medicine, Juntendo University, Tokyo, Japan, *5) Departments of \\ General Medicine, Juntendo University Graduate School of Medicine, Tokyo, Japan, *6) Department of Tropical Medicine and \\ Parasitology, Juntendo University Graduate School of Medicine, Tokyo, Japan, *7) Department of General Thoracic Surgery, \\ Juntendo University Graduate School of Medicine, Tokyo, Japan, *8) Department of Respiratory Medicine, Juntendo University \\ Graduate School of Medicine, Tokyo, Japan, ${ }^{* 9}$ ) Tropical Medicine Association of Juntendo University (TMAJU), Tokyo, \\ Japan
}

The Tropical Medicine Association of Juntendo University (TMAJU) is a student group of Juntendo University founded by students as well as the chief executive officer (CEO) of Juntendo University, Prof. Hideoki Ogawa, nearly 60 years ago. TMAJU has three missions: "Learning beyond the textbook", "Creating ties" and "Emphasizing responding as global medical students". Based on these missions, we have been learning about tropical medicine through daily studying sessions and a study trip to Thailand and Singapore, interacting with medical students in many countries in the South East Asia area. Over a 12-day period, from March 16-27, 2018, the TMAJU 54th South East Asia research team, which consisted of 10 students in their second to fifth years of university and professors from Juntendo University, visited Thailand and Singapore to learn about tropical medicine. We enjoyed valuable experiences learning about tropical medicine and foreign healthcare systems through this trip. We hope that future members of TMAJU will also take part in this great experience and build upon it as years pass.

Key words: Tropical Medicine Association of Juntendo University (TMAJU), leprosy, HIV/AIDS, Tuberculosis (TB), Malaria

\section{Participating members}

Chief: Prof. Kazuhisa Takahashi (Dept. of Respiratory Medicine)

Deputy Chief: Prof. Kenji Suzuki (Dept. of General Thoracic Surgery)

Accompanying faculty: Prof. Toshihiro Mita (Dept. of Tropical Medicine and Parasitology), Dr. Kenji Murai (Dept. of General Medicine)

Student leader: Shohei Fujikawa (4th Year)

Student sub-leader: Tomoki Hongo (4th Year)

Student members: Hana Otani (5th Year), Chikako

Naoe (4th Year), Megumi Hisanaga (3rd year),

Corresponding author: Shohei Fujikawa

The 4th Year Students of Faculty of Medicine, Juntendo University

2-1-1 Hongo, Bunkyo-ku, Tokyo 113-8421, Japan

TEL: +81-3-3813-3111 E-mail: med2114097@stud.juntendo.ac.jp

〔Received Nov. 23, 2018〕 ‘Accepted Jan. 16, 2019〕

J-STAGE Advance published date: Apr. 3, 2019

Copyright (C) 2019 The Juntendo Medical Society. This is an open access article distributed under the terms of Creative Commons Attribution License (CC BY), which permits unrestricted use, distribution, and reproduction in any medium, provided the original source is properly credited. doi: $10.14789 /$ jmj .2019.65.JMJ18-Re02 
Shunya Iijima (2nd Year), Kenta Hikotani (2nd Year), Riho Takeda (2nd Year), Momoko Iijima (2nd Year), Sayuki Ochiai (2nd Year)

\section{Aim}

We, the Tropical Medicine Association of Juntendo University (TMAJU) have been studying tropical medicine for many years, with a particular focus on four tropical diseases: tuberculosis (TB), malaria, leprosy and HIV/AIDS. To prepare for our study trip to Thailand and Singapore, we held study meetings on tropical diseases every month. In addition, we visited several relevant medical facilities in Japan, such as Fukujuji Hospital, which is a national TB hospital; and the National Center for Global Health and Medicine and Okinawa Airakuen, which is a sanitarium for leprosy patients. To help shape our ideas, we visit Singapore and Thailand every spring.

This year, we visited the same facilities as last year in order to examine problems we had noticed on our previous trip in greater detail.

\section{About our study tour}

Singapore

A. National University of Singapore

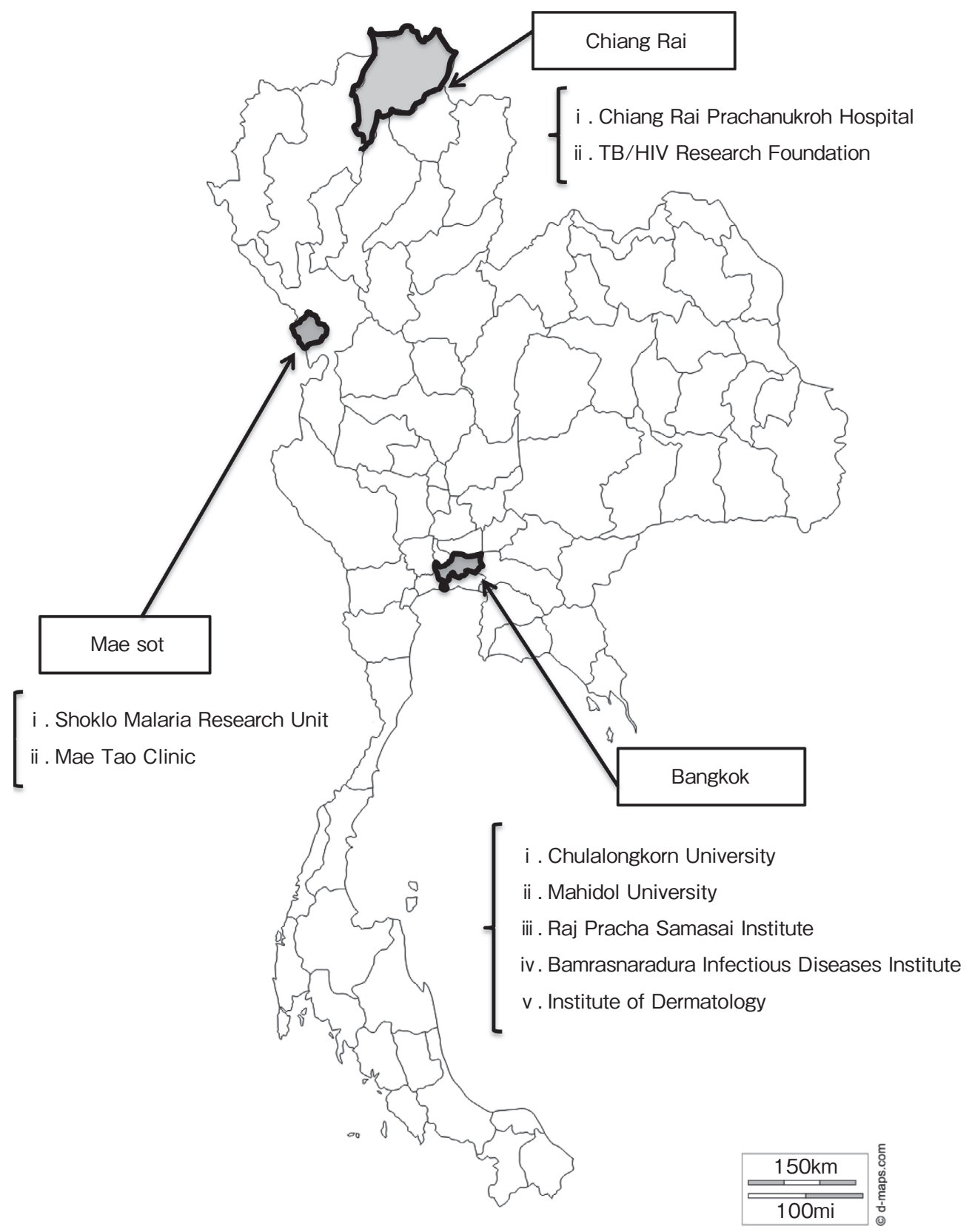

Figure-1 The location of the facilities that we visited in Thailand 


\author{
Thailand (Figure-1) \\ A. Bangkok \\ i . Chulalongkorn University \\ ii. Mahidol University \\ iii. Raj Pracha Samasai Institute (RPSI) \\ iv. Bamrasnaradura Infectious Diseases \\ Institute (BIDI) \\ v. Population and Community Development \\ Association (PDA) \\ iv. Institute of Dermatology (IOD) \\ B. Mae Sot
i. Shoklo Malaria Research Unit (SMRU)
ii. Mae Tao Clinic
i . TB/HIV Research Foundation (THRF)
ii . Chiang Rai Prachanukroh Hospital \\ C. Chiang Rai
}

At the National University of Singapore (NUS), we received a lecture about the anatomy of the palate, which helped us understand inborn deformities, such as palatognathous disease. We also had a look around the anatomical museum and joined a clinical case study class. This was quite an impressive experience, as almost all of the students in the class had positive opinions on both the lecture and case study, because, students listened to the lecture very eagerly, and never stopped discussing the problem each other in case studies. In addition, the students were not afraid to make mistakes, as these contributed to active discussion. We stayed at the home of NUS students, spending much of the duration of our stay in Singapore with them and greatly enjoying interacting with them while discussing their school lives, hobbies, club activities and future goals.

In Bangkok, we visited six facilities: Chulalongkorn University, Mahidol University, the Raj Pracha Samasai Institute (RPSI), the Bamrasnaradura Infectious Diseases Institute (BIDI), the Population and Community Development Association (PDA) and the Institute of Dermatology (IOD). At Chulalongkorn University, we went on a campus tour that included the memorial museum, where we were taught the long history of the university, and the surgical training room, in which orthopedists from other Asian countries practice surgery using cadavers. We were surprised to see orthopedists from Japan studying there. While walking around the campus, we shared our opinions about univer- sity classes and practical training with the Thai medical students.

At Mahidol University, we went on a hospital tour, which included the pediatric and emergency departments. At the pediatric department, we observed outpatients, the neonatal intensive-care unit and an inpatient ward. At the emergency department, we observed emergency outpatients, of whom there were many, and learned about the emergency system in Thailand. Through our annual visits, we have realized that the medical level in Thailand has been improving year by year, and we can learn quite a lot from their efforts. For example, motorcycle-style ambulances can reach patients without being affected by traffic jams, and blood products are available for free to every hospital.

At the RPSI, which specializes in treating leprosy patients, we visited a dispensary. There is a small village located near the hospital. We were informed by the medical staff that leprosy patients lived in this village, helping those who recover to return to a normal life. The RPSI is, as is obvious from its name, a royal institute, and there was a school located next to the dispensary that had been established by the famous King Bhumibol Adulyadej. Although the patients and their family must undergo screening examinations every year, their disease does not disrupt their lives all that substantially, and they are able to walk around the town as if they are perfectly healthy.

At the BIDI, we learned about activities to encourage people to undergo examinations for HIV/AIDS and about the Thai social security system for immigrants from nearby countries, such as Myanmar and Laos. Thai people can receive screening examinations twice a year for free. As a result of such activities, a total of $98 \%$ of people undergo these examinations each year. The Thai social security system helps immigrants receive affordable treatment, which helps prevent diseases afflicting immigrants from spreading.

At the PDA, we learned how Former Deputy Prime Minister Mechai Viravaidya encouraged the widespread availability of condoms, reducing discomfort with these prophylactics by passing them out for free and encouraging youth to interact with them. His ultimate goal was finally achieved with a reduction in the incidence of sexually transmitted 

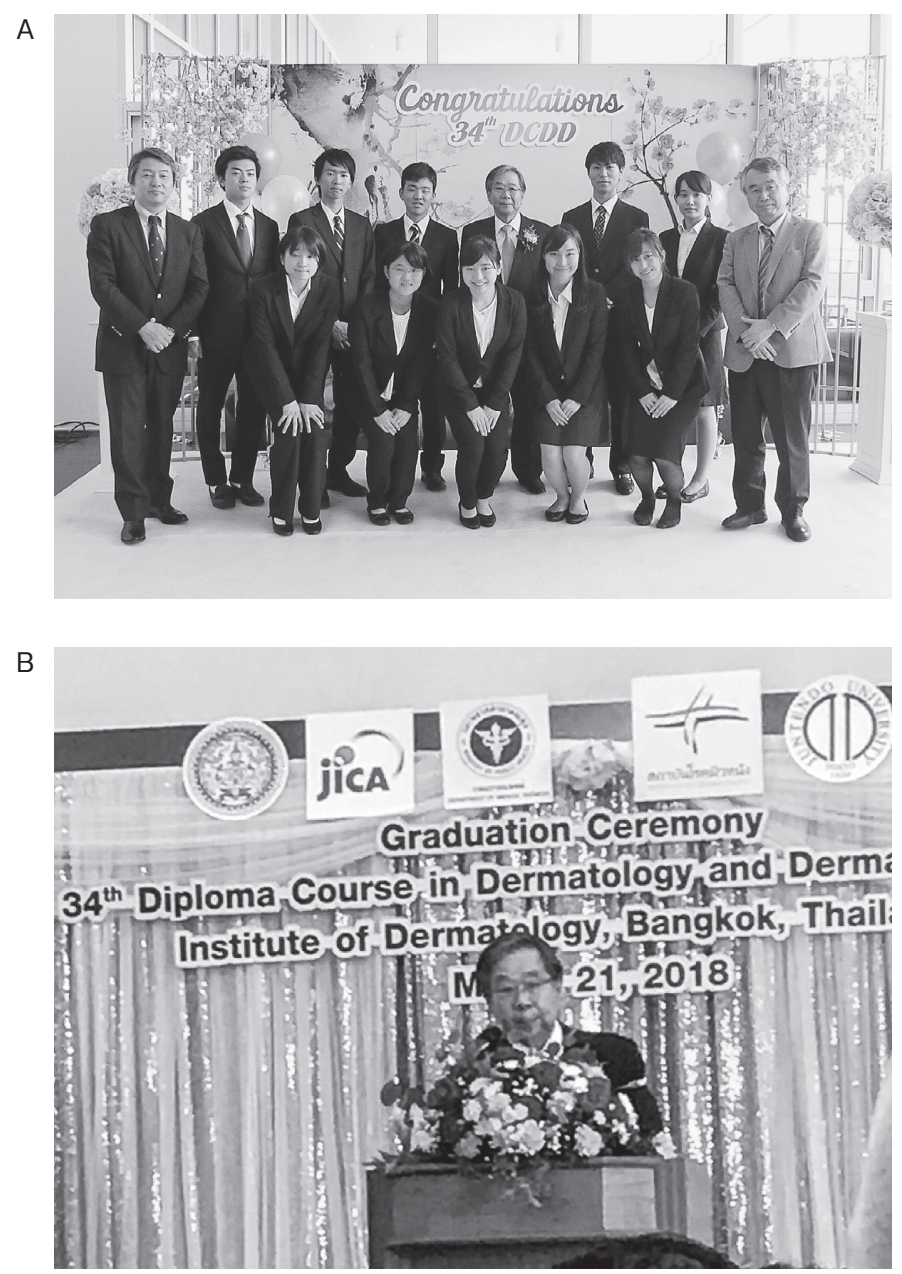

Figure-2 Pictures of students and professors (A) and Prof. Hideoki Ogawa, CEO of Juntendo University (B) at the Reception of the IOD graduation ceremony

infections (STIs) in Thailand. We learned a lesson from this experience concerning measures that can be enacted to cope with the issue of STDs in Japan.

At the IOD, we took part in the graduation ceremony and sang songs for graduates. We are very grateful to be allowed to take part in this ceremony each year thanks to the efforts of Prof. Hideoki Ogawa (Figure-2A, B).

In the latter half of this study trip, we were divided into two teams: one for Mae Sot, the other for Chiang Rai. In Mae Sot, we visited the same two facilities as last year: the Mae Tao Clinic and the Shoklo Malaria Research Unit (SMRU). Mae Sot is located in the northwestern part of Thailand along the border between Thailand and Myanmar. Two major problems face this area: malaria, which is resistant to artemisinin, and the influx of refugees from Myanmar. Thailand's border with Myanmar is defined by the river, which is a source of malaria.
While the Karen people originally lived in the border area, they have been oppressed by the Myanmar military government and now live in the area as refugees without access to social or health services officially approved by the government. To resolve these problems, the Mae Tao clinic, which is run only on donations from non-governmental organizations, provides these refugees with healthcare and medical services and conducts SMRU studies on artemisinin-resistant malaria.

On the first day in Mae Sot, we observed the Mae Tao clinic and received an explanation about their activities. On the second day, we visited the market near the border with Myanmar to assess the situation around the border for ourselves. On the third day, we visited SMRU and gave a presentation on how Japan successfully eradicated malaria, which we chose as our main topic to learn through the last year. This was a great opportunity for us to 
see fields in which international cooperation efforts have been introduced with our own eyes and to deliver a presentation at a facility on the cutting edge of malaria research. This experience will surely be useful for those of us looking to pursue careers in internationally-minded medical professions.

In Chiang Rai, our activities consisted of three parts: participating in a public awareness campaign about TB held by the TB/HIV Research Foundation (THRF), going on a hospital tour and visiting a local aged patients' home. During the campaign, we visited a local technical school. After the staff of the THRF gave a lecture on TB, we conducted a simple opinion survey among the students and teachers in attendance. We wished we could have spoken a little more Thai, as we were unfortunately unable to communicate with the local people using English.

At Chiang Rai Prachanukroh Hospital, we saw the hospital's TB outpatients and took a tour through the laboratory of direct smear examinations. While the hospital used extremely advanced testing equipment that is rarely seen in Japan, people at the hospital were easily exposed to the tubercula bacillus spread by the tuberculosis patients, as only a single pane of glass separated the patients and other uninfected individuals, allowing them to breathe the same air. However, despite some undeveloped aspects of treatment, we were truly shocked at the system used to share patient information. Once test results are obtained, the results are shared through SNS (Social Networking Service), such as LINE, so that all staff in the hospital are made immediately aware of whether the tests were positive or negative. This system is revolutionary, as tuberculosis patients must be isolated as soon as possible. When we traveled with a doctor on house calls, we went around the village by car and met an aged patient. This was a precious opportunity for us to learn how the people live in that area, which helped us consider what might have caused the increase in the number of TB patients. For example, isolating aged patients from others might have exacerbated the TB situation. On this point, though, we feel compelled to add that the local people were extraordinarily kind to us, saying that they were happy to see Japanese people visiting such a rural area. We hope that the situation surrounding them improves in the future.

\section{Future prospects}

Regarding malaria, we will continue to identify themes to study each year and hope to have an opportunity to deliver a presentation on these themes to SMRU, which is engaged in advanced research on malaria. Regarding TB, we will study the annual number of TB patients in Tokyo and the process of their diagnosis and care, after which we will compare the situation in Japan with that of Thailand. We also intend to explore the problems we observed during this spring study visit. Regarding HIV/AIDS, we plan to look into major problems patients have in Japan and then compare the social and medical situations surrounding these patients. We are also interested in whether or not they feel that they have experienced some form of discrimination and want to know what we can do to realize the society comfortable for them. As part of our research, we plan to participate in an activity called Delivery Boys in Shinjuku, Japan, in which participants deliver condoms to ordinary people. This activity is an imitation of the above-mentioned Thai policy. Regarding leprosy, we will review cultural and social problems that patients have experienced thus far and address new issues that have only recently been recognized. We also hope to expand our horizons to address problems associated with leprosy in Thailand as well.

Before the next study visit, we will study the local problems and social background related to the four above-mentioned diseases and consider the differences in these patients' characteristics and treatment between Japan and Thailand.

We plan to visit Maehea as a new destination for the spring study tour in Thailand. We will visit Rajabhat University to learn about public health, Sanpaton Hospital to learn about HIV and TB and Sarapin Hospital to broaden our knowledge about patients who have immigrated from foreign countries. Since this is our first time to visit such spaces, our aim is to identify the social and medical problems and compile questions that we can address on our next visit.

Consequently, we aim to maintain a cosmopolitan outlook to strengthen the networking between domestic and foreign medical institutions. In order to do so, we should not hesitate to communicate with foreigners in our fields and understand the 
finer points of both domestic and foreign medicine. We believe that sharing information on mutual medical fields will help better patients' lives around the world.

\section{Acknowledgement}

We, the TMAJU members have been continuing our studies of tropical diseases and plan to continue with these study visits every year; however, such visits would be impossible without the help of many people.

First, we would like to express our sincere appreciation to Prof. Hideoki Ogawa, CEO of Juntendo University, for his great support. We would like to thank Dr. Hiroshi Tsuda for his voluntary support of our activities over the past few decades. We also would like to thank our local coordinator, Mr. Teruhiko Sekiguchi, who has been of great help to the TMAJU over many years.

We have been able to maintain good relationships in Bangkok thanks to several institutions. Concerning our visit to Chulalongkorn University, we would like to thank Associate Prof. Unnop Jaisamrarn, Associate Dean for International Affairs; Prof. Sittisak Honsawek, Assistant Dean for Student Affairs; and Mr. Watcharapol Malaiwong, The Officer of International Affairs for accepting our study visit. From Mahidol University, we thank Mr. Kitisak Jaijunla for coordinating our visit to the pediatric medicine ward and the emergency ward and Mr. Bhakin Khantarjeerawat for coordinating our visit to Mahidol University.

In addition to these universities, we would also like to thank Mr. Isadore Reaud and Dr. Wolfgang Frank from the PDA, Ms. Suwanna Jarutan from the IOD, Napat Chitwarakorn, M.D. from the BIDI and Dr. Arjin Cholapand and Dr. Naruemol Chaidee from the RPSI.

Our Mae Sot group would like to thank Ms. Naw Annie, Ms. Sophia and Ms. Tsubasa Saito for making our Mae Tao Clinic visit very memorable and Dr. Rose McGready and Ms. Wannee Ritwongsakul for coordinating and organizing our visit to SMRU. Our Chaing Rai group would like to thank Dr. Surasit Bupachat and Mr. Koshiro Nagasaki from the THRF and Dr. Supalert, Dr. Worrarat and Dr. Daranee from Chiang Rai Prachanukroh Hospital for their amazing contributions to our visit.

We also thank Dr. Hideki Yanai from the Japan International Cooperation Agency (JICA), Dr. Norio Ohmagari, Dr. Shinichiro Morioka, Dr. Kenji Kutsuna, Dr. Kunihisa Tsukada, Ms. Sangnim Lee from Center Hospital of The National Center For Global Health and Medicine, Ms. Kyoko Sato from Tama Zenshoen (a sanitarium for leprosy patients in Tokyo), Prof. Toshio Naito, Prof. Tadayuki Yuasa, Dr. Takatoshi Ogawa, Dr. Yuki Uehara and Dr. Tadashi Sato for sharing their precious time in the form of preparatory lectures for our study trip. 